\title{
On the Domain of Analyticity and Small Scales for the Solutions of the Damped-Driven 2D Navier-Stokes Equations
}

\author{
Alexei A. Ilyin and Edriss S. Titi \\ Communicated by Yuncheng You, received January 19, $200 \%$.
}

\begin{abstract}
We obtain a logarithmicaly sharp estimate for the space-analyticity radius of the solutions of the damped-driven 2D Navier-Stokes equations with periodic boundary conditions and relate this to the small scales in this system. This system is inspired by the Stommel-Charney barotropic ocean circulation model.
\end{abstract}

\section{Contents}

1. Introduction 111

2. Gevrey regularity of the damped Navier-Stokes system 114

3. Sharper bounds 117

4. Concluding remarks $\quad 125$

$\begin{array}{ll}\text { Acknowledgments } & 125\end{array}$

References $\quad 126$

\section{Introduction}

It was shown in $[\mathbf{1 7}]$ (see also $[\mathbf{3}],[\mathbf{1 3}]$ ) that the solutions of the 2D NavierStokes equations with periodic boundary conditions belong to the Gevrey class of analytic functions (if the forcing term does). Using the Gevrey regularity approach the following estimate for the spatial analyticity radius for the solutions that lie on the global attractor (or are near it) was obtained

$$
l_{a} \geq \frac{c|\Omega|^{1 / 2}}{G^{2} \log G},
$$

1991 Mathematics Subject Classification. 35B41, 35Q30, 37L30.

Key words and phrases. Analyticity, Gevrey regularity, Navier-Stokes equations, dissipative length scales, Stommel-Charney model. 
where $G=\|f\|_{L_{2}}|\Omega| / \nu^{2}$ is the Grashof number and $|\Omega|=L^{2} / \gamma$ is the area of the periodic domain $\Omega=[0, L / \gamma] \times[0, L], \gamma \leq 1$.

Therefore, the Fourier coefficients $\hat{u}_{k}$ are exponentially small for $|k| \gg L / l_{a}$, and $l_{a}$ naturally forms a lower bound for the small dissipative length scale for the system (see, for instance, $[\mathbf{1 1}]$ ).

There are other ways of estimating the dissipative small length scale for the Navier-Stokes system, for instance, in terms of the dimension of the global attractor $[\mathbf{1}],[\mathbf{6}],[\mathbf{7}],[\mathbf{1 3}],[\mathbf{4 1}]$. The Hausdorff and fractal dimensions of the global attractor satisfy the following estimate $[\mathbf{8}]$ (see also $[\mathbf{6}],[\mathbf{4 1}]$ ):

$$
\operatorname{dim}_{F} \mathcal{A} \leq c_{1} G^{2 / 3}(\log (1+G))^{1 / 3}, \quad c_{1}=c_{1}(\gamma)
$$

which has been shown in [35] (following ideas of [1] ) to be logarithmically sharp. It worth mentioning, however, that for 2D turbulent flows which satisfy the Kraichnan theory of turbulence, that is, a forward enstrophy cascade with power spectrum $k^{-3}$ for the energy density, it has been shown in [12] that the dimension of the attractor is bounded (up to a logarithmic term) by $G^{1 / 2}$.

If we accept the point of view that the small length scale can be defined as follows (see $[\mathbf{7}],[\mathbf{1 3}],[\mathbf{3 8}],[\mathbf{4 1}])$

$$
l_{f} \sim\left(\frac{|\Omega|}{\operatorname{dim}_{F} \mathcal{A}}\right)^{1 / 2},
$$

then up to logarithmic correction we have

$$
l_{f} \sim \frac{|\Omega|^{1 / 2}}{G^{1 / 3}} .
$$

This heuristic estimate for the small length scale is probably the best one can hope for since it matches, up to logarithmic term, the physically asserted estimates for the enstrophy dissipation length scale [32]. We also observe that the estimate (1.3) is extensive, that is, independent of the size of the spatial domain provided that its shape is fixed. We again point out that rigorous estimates in [15] suggest that the enstrophy dissipation length scale $l_{d}$ satisfies the estimate

$$
c^{\prime}|\Omega|^{1 / 2} G^{-1 / 3} \leq l_{d} \leq c^{\prime \prime}|\Omega|^{1 / 2} G^{-1 / 6}
$$

for 2D the turbulent flows that satisfy the Kraichnan theory of turbulence.

Another rigorous definition of the small length scale can be given in terms of the number of determining modes, nodes, or volume elements (see [13], [16], [18], $[30]$ and the references therein). It was shown that if $N$ is sufficiently large and $N$ equal squares of size $l_{d n}$ tile the periodic spatial domain, then any collection of points (one in each square) are determining for the long time dynamics of the $2 \mathrm{D}$ Navier-Stokes system. The best to date estimate for $N$ was obtained in [30]:

$$
N \leq c_{2} G
$$

where $c_{2}=c_{2}(\gamma)$ depends only on the aspect ratio $\gamma \leq 1$. (An explicit estimate for $c_{2}$ was obtained in $[\mathbf{2 8}]: c_{2}(\gamma)=(68 /(\gamma \pi))^{1 / 2}$.)

Therefore the small length scale defined in terms of the lattice of determining nodes satisfies

$$
l_{d n} \geq c_{2}(\gamma)^{-1 / 2} \frac{|\Omega|^{1 / 2}}{G^{1 / 2}} .
$$


We observe that this estimate is not extensive, that is, $l_{d n}$ scales like $\lambda^{-1 / 2}$ if $\Omega$ is replaced by $\lambda \Omega, \lambda>0$.

We point out here that for the 2D Navier-Stokes system with analytic forcing the results of $[\mathbf{1 9}],[\mathbf{2 0}]$ provide the existence of a finite number $N$ of instantaneously determining nodes comparable with the fractal dimension of the attractor. These nodes, however, can be chosen arbitrarily (up to a subset of $\Omega^{N}$ with $2 N$ dimensional Lebesgue measure zero) and therefore do not naturally define a regular lattice of determining nodes.

The best to date estimate for the analyticity radius of the solutions of the Navier-Stokes equations with analytic forcing term $f$ was obtained in [33]:

$$
l_{a} \geq c_{3}(\gamma) \frac{|\Omega|^{1 / 2}}{G^{1 / 2}(1+\log G)^{1 / 4}} .
$$

Relating the radius of analyticity to the dissipative small length scale (see also [25] in this regard) we note that up to a logarithmic correction the estimate (1.5) coincides with (1.4), but both are worse than (1.2), where the latter coincides, as we have already pointed out, with the physically asserted estimate of [32].

In this paper we focus on the $2 \mathrm{D}$ space periodic Navier-Stokes system with damping

$$
\begin{aligned}
\partial_{t} u+\sum_{i=1}^{2} u^{i} \partial_{i} u & =-\mu u+\nu \Delta u-\nabla p+f \\
\operatorname{div} u & =0
\end{aligned}
$$

By adding the Coriolis forcing term to (1.6) one obtains the well-known StommelCharney barotropic model of ocean circulation $[4],[\mathbf{1 0}],[\mathbf{3 7}]$, [39]. Here the damping $\mu u$ represents the Rayleigh friction term and $f$ is the wind stress. For an analytical study of this system see, for instance $[\mathbf{5}],[\mathbf{2 4}],[\mathbf{2 6}],[\mathbf{4 3}]$, and the references therein. In a follow up work we will be studying the effect of adding rotation (Coriolis parameter) on the size of small scales and the complexity of the dynamics of (1.6). Therefore, we will focus in this work on the system (1.6). We also point out that in this geophysical context the viscosity plays a much smaller role in the mechanism of dissipating energy than the Rayleigh friction. That is why in this work the friction coefficient $\mu>0$ will be fixed and we consider the system at the limit when $\nu \rightarrow 0^{+}$.

Sharp estimates (as $\nu \rightarrow 0$ ) for the Hausdorff and the fractal dimensions of the global attractor of the system (1.6) were first obtained in the case of the squareshaped domain in $[\mathbf{2 7}](\gamma=1)$. Then the case of an elongated domain was studied in $[\mathbf{2 9}](\gamma \rightarrow 0)$, where it was shown that

$$
\operatorname{dim}_{F} \mathcal{A} \leq c_{4} D, \quad D=\frac{\|\operatorname{rot} f\|_{\infty}|\Omega|}{\mu \nu},
$$

where $c_{4}$ is an absolute constant $\left(c_{4} \leq 12\right)$. This estimate is sharp as both $\nu \rightarrow 0$ and $\gamma \rightarrow 0$. Therefore the small length scale defined as in (1.2) is of the order of

$$
l_{f} \sim\left(\frac{|\Omega|}{\operatorname{dim}_{F} \mathcal{A}}\right)^{1 / 2} \sim\left(\frac{\mu \nu}{\|\operatorname{rot} f\|_{\infty}}\right)^{1 / 2} \sim \frac{|\Omega|^{1 / 2}}{D^{1 / 2}} .
$$


This heuristic estimate is, in fact, a rigorous bound for the small length scale expressed in terms of the number of determining modes and nodes [28]:

$$
l_{d n}=c_{5}\left(\frac{|\Omega|}{D}\right)^{1 / 2}=c_{5}\left(\frac{\mu \nu}{\|\operatorname{rot} f\|_{\infty}}\right)^{1 / 2}, \quad c_{5}=68^{1 / 4} .
$$

This means that any lattice of points in $\Omega$ at a typical distance $l \leq l_{d n}$ is determining.

The main result of this paper is in showing that the analyticity radius $l_{a}$ of the solutions of the damped-driven Navier-Stokes system (1.6) lying on the global attractor is bounded from below and satisfies the estimate:

$$
l_{a} \geq \frac{c|\Omega|^{1 / 2}}{D^{1 / 2}(1+\log D)^{1 / 2}},
$$

which up to a logarithmic correction agrees both with the smallest scale estimate (1.8) and the rigorously defined typical distance between the determining nodes (1.9).

It is worth mentioning that this point of view of relating the radius of analyticity of solutions on the Navier-Stokes equations to small scales in turbulence was also presented in $[\mathbf{2 5}]$.

This paper is organized as follows. In section 2 we employ the Gevrey-Hilbert space technique of $[\mathbf{1 7}]$ to derive a lower bound for the radius of analyticity of the order

$$
\frac{c|\Omega|^{1 / 2}}{D^{2} \log D}
$$

This bound considerably improves, for a fixed $\mu>0$, the lower bound (1.1) for the classical Navier-Stokes system as $\nu \rightarrow 0^{+}$(see also Remark 2.1). Let us remark that as an alternative to the Gevrey regularity technique for estimating small scales one can apply the ladder estimates approach presented in $[\mathbf{9}]$ to obtain estimates for the small scales in (1.6) (see also [21]).

In section 3 the estimate (1.10) is proved for the system (1.6) following [33].

\section{Gevrey regularity of the damped Navier-Stokes system}

As usual (see, for instance, $[\mathbf{1}],[\mathbf{6}],[\mathbf{3 4}],[\mathbf{4 0}]$ ), we write (1.6) as an evolution equation in the Hilbert space $H$ which is the closed subspace of solenoidal vectors in $\left(L_{2}(\Omega)\right)^{2}$ with zero average over the torus $\Omega=[0, L / \gamma] \times[0, L]$ :

$$
\partial_{t} u+B(u, u)+\nu A u+\mu u=f, \quad u(0)=u_{0} .
$$

Here $A=-P \Delta$ is the Stokes operator with eigenvalues $0<\lambda_{1} \leq \lambda_{2} \leq \ldots$, $B(u, v)=P\left(\sum_{i=1}^{2} u^{i} \partial_{i} v\right)$ is the nonlinear term, $f=P f \in H$, and $P:\left(L_{2}(\Omega)\right)^{2} \rightarrow$ $H$.

We restrict ourselves to the case $\gamma=1$ and, in addition, assume that $\Omega=$ $[0,2 \pi]^{2}$ (this simplifies the Fourier series below). The case of the square-shaped domain $\Omega=[0, L]^{2}$ reduces to this case by scaling. Furthermore, any domain with aspect ratio $\gamma<1$ can be treated in the similar way, the absolute dimensionless constants $c_{1}, c_{2}, \ldots$ below will then depend on $\gamma$, however.

A vector field $u \in H$ has the Fourier series expansion

$$
u=\sum_{j \in \mathbb{Z}^{2}} u_{j} e^{i j \cdot x}, \quad u_{j} \in \mathbb{C}^{2}, \quad u_{-j}=\bar{u}_{j}, \quad u_{j} \cdot j=0, \quad u_{0}=0,
$$


and

$$
\|u\|^{2}=\|u\|_{L_{2}}^{2}=(2 \pi)^{2} \sum_{j \in \mathbb{Z}^{2}}\left|u_{j}\right|^{2} .
$$

The eigenvalues of the Stokes operator $A$ are the numbers $|j|^{2}$, and the domain of its powers is the set of vector functions $u$ such that

$$
(2 \pi)^{2} \sum_{j \in \mathbb{Z}^{2}}|j|^{4 \alpha}\left|u_{j}\right|^{2}=\left\|A^{\alpha} u\right\|^{2}<\infty .
$$

For $\tau, s>0$ we define the Gevrey space $D\left(e^{\tau A^{s}}\right)$ of functions $u$ satisfying

$$
(2 \pi)^{2} \sum_{j \in \mathbb{Z}^{2}} e^{2 \tau|j|^{2 s}}\left|u_{j}\right|^{2}=\left\|e^{\tau A^{s}} u\right\|^{2}<\infty .
$$

We suppose that the forcing term $f$ belongs to the Gevrey space of analytic functions

$$
f \in D\left(e^{\sigma_{1} A^{1 / 2}} A^{1 / 2}\right),
$$

so that

$$
(2 \pi)^{2} \sum_{j \in \mathbb{Z}^{2}}|j|^{2} e^{2 \sigma_{1}|j|}\left|u_{j}\right|^{2}=\left\|e^{\sigma_{1} A^{1 / 2}} A^{1 / 2} f\right\|<\infty
$$

for some $\sigma_{1}>0$. We set

$$
\varphi(t)=\min \left(\nu \lambda_{1}^{1 / 2} t, \sigma_{1}\right) .
$$

The norm and the scalar product in $D\left(e^{\varphi(t) A^{1 / 2}}\right)$ are denoted by $\|\cdot\|_{\varphi}$ and $(\cdot, \cdot)_{\varphi}$, respectively.

We assume that $u_{0} \in D\left(A^{1 / 2}\right)$ and take the scalar product of (2.1) and $A u$ in $D\left(e^{\varphi(t) A^{1 / 2}}\right)$ for sufficiently small $t \leq \sigma_{1} /\left(\nu \lambda_{1}^{1 / 2}\right)$. Since

$$
\left(e^{\varphi(t) A^{1 / 2}} \partial_{t} u(t), e^{\varphi(t) A^{1 / 2}} A u(t)\right)=\frac{1}{2} \partial_{t}\left\|A^{1 / 2} u(t)\right\|_{\varphi}^{2}-\nu \lambda_{1}^{1 / 2}\left(A u(t), A^{1 / 2} u(t)\right)_{\varphi},
$$

we obtain

$$
\begin{aligned}
& \frac{1}{2} \partial_{t}\left\|A^{1 / 2} u\right\|_{\varphi}^{2}+\nu\|A u\|_{\varphi}^{2}+\mu\left\|A^{1 / 2} u\right\|_{\varphi}^{2} \\
& =-(B(u, u), A u)_{\varphi}+\nu \lambda_{1}^{1 / 2}\left(A u, A^{1 / 2} u\right)_{\varphi}+\left(A^{1 / 2} f, A^{1 / 2} u\right)_{\varphi} .
\end{aligned}
$$

Next we use the key estimate (see $[\mathbf{1 7}],[\mathbf{1 3}],[\mathbf{4 2}]$ ) for the nonlinear term in Gevrey spaces

$$
|(B(u, u), A u)|_{\varphi} \leq c_{1}\left\|A^{1 / 2} u\right\|_{\varphi}^{2}\|A u\|_{\varphi}\left(1+\log \frac{\|A u\|_{\varphi}^{2}}{\lambda_{1}\left\|A^{1 / 2} u\right\|_{\varphi}^{2}}\right)^{1 / 2}
$$

and use Young's inequality for this estimate and for the last two terms in (2.4):

$$
\begin{aligned}
\partial_{t}\left\|A^{1 / 2} u\right\|_{\varphi}^{2}+\nu & \|A u\|_{\varphi}^{2} \\
& \leq \frac{2 c_{1}^{2}}{\nu}\left\|A^{1 / 2} u\right\|_{\varphi}^{4}\left(1+\log \frac{\|A u\|_{\varphi}^{2}}{\lambda_{1}\left\|A^{1 / 2} u\right\|_{\varphi}^{2}}\right)+2 \nu \lambda_{1}\left\|A^{1 / 2} u\right\|_{\varphi}^{2}+\frac{\left\|A^{1 / 2} f\right\|_{\varphi}^{2}}{2 \mu} \\
& \leq \frac{c_{2}}{\nu}\left\|A^{1 / 2} u\right\|_{\varphi}^{4}\left(1+\log \frac{\|A u\|_{\varphi}^{2}}{\lambda_{1}\left\|A^{1 / 2} u\right\|_{\varphi}^{2}}\right)+\nu^{3} \lambda_{1}^{2}+\frac{\left\|A^{1 / 2} f\right\|_{\varphi}^{2}}{2 \mu},
\end{aligned}
$$


where $c_{2}=2 c_{1}^{2}+1$. As in [13], [14] we use the inequality $-\alpha z+\beta(1+\log z) \leq$ $\beta \log \beta / \alpha$ to find that

$$
-\nu\|A u\|_{\varphi}^{2}+\frac{c_{2}}{\nu}\left\|A^{1 / 2} u\right\|_{\varphi}^{4}\left(1+\log \frac{\|A u\|_{\varphi}^{2}}{\lambda_{1}\left\|A^{1 / 2} u\right\|_{\varphi}^{2}}\right) \leq \frac{c_{2}}{\nu}\left\|A^{1 / 2} u\right\|_{\varphi}^{4} \log \frac{c_{2}\left\|A^{1 / 2} u\right\|_{\varphi}^{2}}{\lambda_{1} \nu^{2}},
$$

and obtain the differential inequality

$$
\partial_{t}\left\|A^{1 / 2} u\right\|_{\varphi}^{2} \leq \frac{c_{2}}{\nu}\left\|A^{1 / 2} u\right\|_{\varphi}^{4} \log \frac{c_{2}\left\|A^{1 / 2} u\right\|_{\varphi}^{2}}{\lambda_{1} \nu^{2}}+\nu^{3} \lambda_{1}^{2}+\frac{\left\|A^{1 / 2} f\right\|_{\varphi}^{2}}{2 \mu} .
$$

Hence the function

$$
y(t)=\frac{c_{2}\left\|A^{1 / 2} u\right\|_{\varphi}^{2}}{\lambda_{1} \nu^{2}}+\frac{\left\|A^{1 / 2} f\right\|_{\sigma_{1}}}{\lambda_{1} \nu^{3 / 2} \mu^{1 / 2}}+e,
$$

where $\ln e=1$, satisfies

$$
\partial_{t} y(t) \leq \nu \lambda_{1} c_{3} y^{2} \log y, \quad c_{3}=\max \left(1, c_{2} / 2\right) .
$$

If $y(t) \leq 2 y(0)$, then $\partial_{t} y(t) \leq \nu \lambda_{1} c_{3} y^{2} \log (2(y(0))$. Hence we indeed have $y(t) \leq$ $2 y(0)$ for

$$
t \leq\left(2 \nu \lambda_{1} c_{3} y(0) \log (2 y(0))\right)^{-1} .
$$

In other words,

$$
\left\|A^{1 / 2} u\right\|_{\varphi}^{2} \leq 2\left\|A^{1 / 2} u_{0}\right\|^{2}+c_{4}(\nu / \mu)^{1 / 2}\left\|A^{1 / 2} f\right\|_{\sigma_{1}}+c_{4} \lambda_{1} \nu^{2}, \quad c_{4}=e / c_{2},
$$

as long as $0 \leq t \leq T^{*}\left(\left\|A^{1 / 2} u_{0}\right\|\right)$, where

$$
\begin{aligned}
& T^{*}\left(\left\|A^{1 / 2} u_{0}\right\|\right)= \\
& 2 c_{3} \nu \lambda_{1}\left(\frac{c_{2}\left\|A^{1 / 2} u_{0}\right\|^{2}}{\lambda_{1} \nu^{2}}+\frac{\left\|A^{1 / 2} f\right\|_{\sigma_{1}}}{\lambda_{1} \nu^{3 / 2} \mu^{1 / 2}}+e\right) \log \left(2\left(\frac{c_{2}\left\|A^{1 / 2} u_{0}\right\|^{2}}{\lambda_{1} \nu^{2}}+\frac{\left\|A^{1 / 2} f\right\|_{\sigma_{1}}}{\lambda_{1} \nu^{3 / 2} \mu^{1 / 2}}+e\right)\right)
\end{aligned}
$$

We now observe (see Lemma 3.1) that on the global attractor or in the absorbing ball we have, respectively,

$$
\left\|A^{1 / 2} u(t)\right\| \leq \frac{\left\|A^{1 / 2} f\right\|}{\mu}, \quad t \in \mathbb{R}, \quad\left\|A^{1 / 2} u(t)\right\| \leq 2 \frac{\left\|A^{1 / 2} f\right\|}{\mu}, \quad t \geq T_{0}\left(\left\|A^{1 / 2} u_{0}\right\|\right) .
$$

Therefore we have the following lower bound for $T^{*}$ :

$T^{*} \geq c_{5}\left[\nu \lambda_{1}\left(\frac{\left\|A^{1 / 2} f\right\|^{2}}{\lambda_{1} \nu^{2} \mu^{2}}+\frac{\left\|A^{1 / 2} f\right\|_{\sigma_{1}}}{\lambda_{1} \nu^{3 / 2} \mu^{1 / 2}}+1\right) \log \left(\frac{\left\|A^{1 / 2} f\right\|^{2}}{\lambda_{1} \nu^{2} \mu^{2}}+\frac{\left\|A^{1 / 2} f\right\|_{\sigma_{1}}}{\lambda_{1} \nu^{3 / 2} \mu^{1 / 2}}+1\right)\right]^{-1}$

In the limit $\nu \rightarrow 0^{+}$we have

$$
\frac{\left\|A^{1 / 2} f\right\|^{2}}{\lambda_{1} \nu^{2} \mu^{2}} \gg \frac{\left\|A^{1 / 2} f\right\|_{\sigma_{1}}}{\lambda_{1} \nu^{3 / 2} \mu^{1 / 2}}
$$

and we can write the lower bound for $T^{*}$ as follows

$$
T^{*} \geq c_{6}\left[\nu \lambda_{1} D^{2} \log D\right]^{-1},
$$

where

$$
\frac{\left\|A^{1 / 2} f\right\|}{\lambda_{1}^{1 / 2} \nu \mu}=\frac{\|\operatorname{rot} f\||\Omega|^{1 / 2}}{2 \pi \nu \mu} \leq \frac{1}{2 \pi} D, \quad \text { where } \quad D=\frac{\|\operatorname{rot} f\|_{\infty}|\Omega|}{\nu \mu} .
$$

In terms of the analyticity radius $l_{a}$ the lower bound for $T^{*}$ takes the form

$$
l_{a} \geq \frac{c_{7}|\Omega|^{1 / 2}}{D^{2} \log D} .
$$


Thus, we have proved the following theorem.

THEOREM 2.1. Suppose that $f \in D\left(A^{1 / 2} e^{\sigma_{1} A^{1 / 2}}\right)$ for some $\sigma_{1}>0$. Then a solution $u$ lying on the global attractor $\mathcal{A}$ is analytic with analyticity radius

$$
l_{a} \geq \min \left(\frac{c_{7}|\Omega|^{1 / 2}}{\left(D^{2}+D_{1}+1\right) \log \left(D^{2}+D_{1}+1\right)}, \sigma_{1}\right),
$$

where

$$
D=\frac{\|\operatorname{rot} f\|_{\infty}|\Omega|}{\nu \mu}, \quad D_{1}=\frac{\left\|A^{1 / 2} f\right\|_{\sigma_{1}}}{\lambda_{1} \nu^{3 / 2} \mu^{1 / 2}}
$$

Moreover,

$$
l_{a} \geq \frac{c_{8}|\Omega|^{1 / 2}}{D^{2} \log D} \quad \text { as } \quad \nu \rightarrow 0^{+} .
$$

The constants $c_{7}$ and $c_{8}$ depend only on the aspect ratio of the periodic domain $\Omega$.

Remark 2.1. We observe that the estimate (2.5) for the system (1.6) is of the order $\nu^{2} / \log (1 / \nu)$ as far as the dependence on $\nu \rightarrow 0^{+}$is concerned, while the estimate (1.1) for the classical Navier-Stokes system is, in this respect much smaller; namely, is of the order $\nu^{4} / \log (1 / \nu)$.

However, the estimate (2.5) is not sharp and will be improved in the next section to the order of $\nu^{1 / 2} / \log ^{1 / 2}(1 / \nu)$. As has been demonstrated in $[36]$ the Gevrey-Hilbert space technique does not always provide sharp estimates for the radius of analyticity. The mechanism explaining this has been reported in [36] by means of an explicitly solvable model equation.

\section{Sharper bounds}

In this section we obtain sharper lower bounds for the analyticity radius $l_{a}$. This is achieved by combining the $\nu$-independent estimate for the vorticity contained in the following lemma and the $L_{p}$-technique developed in [23], $[\mathbf{3 3}]$ for the uniform analyticity radius of the solutions of the Navier-Stokes equations. We observe that a similar technique has been established earlier in [2] for studying the analyticity of the Euler equations.

Applying the operator rot to (1.6) we obtain the well-known scalar vorticity equation

$$
\partial_{t} \omega+u \cdot \nabla \omega=\nu \Delta \omega-\mu \omega+F,
$$

where $\omega=\operatorname{rot} u, F=\operatorname{rot} f, u=\nabla^{\perp} \Delta^{-1} \omega$, so that $u \cdot \nabla \omega=\nabla^{\perp} \Delta^{-1} \omega \cdot \nabla \omega=$ $J\left(\Delta^{-1} \omega, \omega\right)$, and $\nabla^{\perp}=\left(-\partial_{2}, \partial_{1}\right), J(a, b)=\nabla^{\perp} a \cdot \nabla b$.

LEMma 3.1. (See [28].) The solutions $u(t)$ lying on the global attractor $\mathcal{A}$ satisfy the following bound:

$$
\|\omega(t)\|_{L_{2 k}} \leq \frac{\|\operatorname{rot} f\|_{L_{2 k}}}{\mu}, \quad t \in \mathbb{R}
$$

where $1 \leq k \leq \infty$.

ProOF. We use the vorticity equation (3.1) and take the scalar product with $\omega^{2 k-1}$, where $k \geq 1$ is integer, and use the identity

$$
\left(J(\psi, \varphi), \varphi^{2 k-1}\right)=(2 k)^{-1} \int J\left(\psi, \varphi^{2 k}\right) d x=(2 k)^{-1} \int \operatorname{div}\left(\varphi^{2 k} \nabla^{\perp} \psi\right) d x=0 .
$$


We obtain

$$
\begin{array}{r}
\|\omega\|_{L_{2 k}}^{2 k-1} \partial_{t}\|\omega\|_{L_{2 k}}+(2 k-1) \nu \int|\nabla \omega|^{2} \omega^{2 k-2} d x+\mu\|\omega\|_{L_{2 k}}^{2 k}= \\
=\left(\operatorname{rot} f, \omega^{2 k-1}\right) \leq\|\operatorname{rot} f\|_{L_{2 k}}\|\omega\|_{L_{2 k}}^{2 k-1} .
\end{array}
$$

Hence, by Gronwall's inequality

$$
\|\omega(t)\|_{L_{2 k}} \leq\|\omega(0)\|_{L_{2 k}} e^{-\mu t}+\mu^{-1}\|\operatorname{rot} f\|_{L_{2 k}}\left(1-e^{-\mu t}\right),
$$

and passing to the limit as $k \rightarrow \infty$ we find

$$
\|\omega(t)\|_{\infty} \leq\|\omega(0)\|_{\infty} e^{-\mu t}+\mu^{-1}\|\operatorname{rot} f\|_{\infty}\left(1-e^{-\mu t}\right) .
$$

Now, we let $t \rightarrow \infty$ in the above inequalities and obtain

$$
\limsup _{t \rightarrow \infty}\|\omega(t)\|_{L_{2 k}} \leq \frac{\|\operatorname{rot} f\|_{L_{2 k}}}{\mu}, \quad 1 \leq k \leq \infty,
$$

which gives (3.2) since the solutions lying on the attractor are bounded for $t \in$ $\mathbb{R}$.

As before we consider the square-shaped domain $\Omega=[0, L]^{2}$ and it is now convenient to write (1.6) in dimensionless form. We introduce dimensionless variables $x^{\prime}, t^{\prime}, u^{\prime}$ and $p^{\prime}$ by setting

$$
x=L x^{\prime}, \quad t=\left(L^{2} / \nu\right) t^{\prime}, \quad u=(\nu / L) u^{\prime}, \quad p=\left(\nu^{2} / L^{2}\right) p^{\prime}, \quad \mu=\left(\nu / L^{2}\right) \mu^{\prime} .
$$

We obtain

$$
\begin{aligned}
\partial_{t^{\prime}} u^{\prime}+\sum_{i=1}^{2} u^{\prime i} \partial_{i}^{\prime} u^{\prime} & =-\mu^{\prime} u+\Delta^{\prime} u^{\prime}-\nabla^{\prime} p^{\prime}+f^{\prime}, \\
\operatorname{div}^{\prime} u^{\prime} & =0
\end{aligned}
$$

where $x^{\prime} \in \Omega^{\prime}=[0,1]^{2}, f^{\prime}=\left(L^{3} / \nu^{2}\right) f$. Accordingly, the dimensionless form of (3.1) is as follows (we omit the primes):

$$
\partial_{t} \omega+u \cdot \nabla \omega=\Delta \omega-\mu \omega+F .
$$

Remark 3.1. For dimensionless variables $u^{\prime}$ and $\omega^{\prime}$ the estimate (3.2) with $k=\infty$ takes the form

$$
\left\|\omega^{\prime}\right\|_{\infty}=\left\|\operatorname{rot}^{\prime} u^{\prime}\right\|_{\infty} \leq \frac{\left\|\operatorname{rot}^{\prime} f^{\prime}\right\|_{\infty}}{\mu^{\prime}}=\frac{\|\operatorname{rot} f\|_{\infty} L^{2}}{\nu \mu}=D .
$$

The next lemma is similar to the main estimate for the space analyticity radius in $[33]$.

Lemma 3.2. Suppose that $F$ is a restriction to $\Omega$ (that is, $y=0$ ) of a bounded $x$-periodic analytic function $F(x+i y)+i G(x+i y)$ in the region $|y| \leq \delta_{F}$ and

$$
M_{F}^{2}=\sup _{x \in \Omega,|y| \leq \delta_{F}}\left(F(x+i y)^{2}+G(x+i y)^{2}\right) .
$$

Let $p \geq 3 / 2$ and let

$$
t_{0}=\frac{M_{2 p}^{2}}{C M_{F}^{2} / \mu} .
$$


Here (and throughout) $C$ is a sufficiently large universal constant and $M_{2 p} \geq$ $\left\|\omega_{0}\right\|_{L_{2 p}}$. Then the solution $\omega(t)$ is analytic for $t>0$ and for $0<t \leq t_{0}$ the space analyticity radius of $\omega(t)$ is greater than

$$
\delta(t)=\min \left(\frac{t^{1 / 2}}{C}, \frac{1}{C p t^{(2 p-3) / 4 p} M_{2 p}}, \frac{1}{C p t^{(2 p-3) /(4 p+6)} M_{2 p}^{2 p /(2 p+3)}}, \frac{1}{p t^{1 / 2} M_{2 p}}, \delta_{F}\right) .
$$

Proof. We solve (3.4) by a sequence of approximating solutions (see [31], [33]). We set $u^{(0)}=0$ and $\omega^{(0)}=0$. Then for $\omega^{(n)}, u^{(n)}$ we have the equation

$$
\begin{gathered}
\partial_{t} \omega^{(n)}-\Delta \omega^{(n)}+u^{(n-1)} \cdot \nabla \omega^{(n)}+\mu \omega^{(n)}=F \\
\omega^{(n)}(0)=\omega_{0}=\operatorname{rot} u_{0}, \quad u^{(n)}=\nabla^{\perp} \Delta^{-1} \omega^{(n)} .
\end{gathered}
$$

The solutions $\omega^{(n)}$ and $u^{(n)}$ for $t>0$ have analytic extensions $\omega^{(n)}+i \theta^{(n)}$ and $u^{(n)}+i v^{(n)}$ and since the system (3.7) is linear, their analyticity radius is at least $\delta_{F}$. They satisfy the equation

$$
\begin{aligned}
& \partial_{t}\left(\omega^{(n)}+i \theta^{(n)}\right)-\Delta\left(\omega^{(n)}+i \theta^{(n)}\right) \\
& +\left(u^{(n-1)}+i v^{(n-1)}\right) \cdot \nabla\left(\omega^{(n)}+i \theta^{(n)}\right)+\mu\left(\omega^{(n)}+i \theta^{(n)}\right)=F+i G,
\end{aligned}
$$

or, equivalently, the system

$$
\begin{aligned}
& \partial_{t} \omega^{(n)}-\Delta \omega^{(n)}+\mu \omega^{(n)}+u^{(n-1)} \cdot \nabla \omega^{(n)}-v^{(n-1)} \cdot \nabla \theta^{(n)}=F, \\
& \partial_{t} \theta^{(n)}-\Delta \theta^{(n)}+\mu \theta^{(n)}+u^{(n-1)} \cdot \nabla \theta^{(n)}+v^{(n-1)} \cdot \nabla \omega^{(n)}=G,
\end{aligned}
$$

where, as before, $u^{(n)}=\nabla^{\perp} \Delta^{-1} \omega^{(n)}, v^{(n)}=\nabla^{\perp} \Delta^{-1} \theta^{(n)}$, and the differential operators are taken with respect to $x$. In view of the analyticity of the solutions we have the Cauchy-Riemann equations

$$
\begin{aligned}
\frac{\partial \omega^{(n)}}{\partial y_{j}} & =-\frac{\partial \theta^{(n)}}{\partial x_{j}}, \\
\frac{\partial \omega^{(n)}}{\partial x_{j}} & =\frac{\partial \theta^{(n)}}{\partial y_{j}}, \quad j=1,2,
\end{aligned}
$$

and the similar equations for $u^{(n)}$ and $v^{(n)}$.

Let $\varepsilon>0$. We consider the functional

$$
\psi_{n}(t)=\int_{0}^{1} \int_{\Omega}\left(\omega^{(n)}(x, \alpha t s, t)^{2}+\theta^{(n)}(x, \alpha t s, t)^{2}+\varepsilon\right)^{p} d x d s
$$

We also set

$$
Q_{n}(x, s, t)=\omega^{(n)}(x, \alpha t s, t)^{2}+\theta^{(n)}(x, \alpha t s, t)^{2}+\varepsilon .
$$

Here $t \in \mathbb{R}^{+}$and $\alpha \in \mathbb{R}^{2}$. The combination $\alpha$ ts will play the role of the variable $y$; $p \geq 3 / 2$, and $\varepsilon>0$ is arbitrary.

We differentiate $\psi_{n}(t)$ taking into account (3.8) and use the Cauchy-Riemann equations (3.9) to handle the derivatives with respect to $y$. We obtain

$$
\frac{1}{2 p} \partial_{t} \psi_{n}(t)+I_{0}=I_{1}+I_{2}+I_{3}+I_{4},
$$


where

$$
\begin{array}{r}
I_{0}=\int_{0}^{1} \int_{\Omega} Q_{n}^{p-1}\left(\left|\nabla \omega^{(n)}\right|^{2}+\left|\nabla \theta^{(n)}\right|^{2}+\mu\left(\omega^{(n)}\right)^{2}+\mu\left(\theta^{(n)}\right)^{2}\right) d x d s+ \\
+2(p-1) \int_{0}^{1} \int_{\Omega} Q_{n}^{p-2}\left(\omega^{(n)} \nabla \omega^{(n)}+\theta^{(n)} \nabla \theta^{(n)}\right) \cdot\left(\omega^{(n)} \nabla \omega^{(n)}+\theta^{(n)} \nabla \theta^{(n)}\right) d x d s
\end{array}
$$

and

$$
\begin{aligned}
& I_{1}=\int_{0}^{1} \int_{\Omega} Q_{n}^{p-1}\left(-\omega^{(n)} \nabla \theta^{(n)}+\theta^{(n)} \nabla \omega^{(n)}\right) \cdot \alpha s d x d s, \\
& I_{2}=\int_{0}^{1} \int_{\Omega} Q_{n}^{p-1}\left(\omega^{(n)} \nabla \omega^{(n)}+\theta^{(n)} \nabla \theta^{(n)}\right) \cdot u^{(n-1)} d x d s, \\
& I_{3}=\int_{0}^{1} \int_{\Omega} Q_{n}^{p-1}\left(\omega^{(n)} \nabla \theta^{(n)}-\theta^{(n)} \nabla \omega^{(n)}\right) \cdot v^{(n-1)} d x d s, \\
& I_{4}=\int_{0}^{1} \int_{\Omega} Q_{n}^{p-1}\left(\omega^{(n)} F+\theta^{(n)} G\right) d x d s .
\end{aligned}
$$

The arguments of $Q_{n}$ are $x, s, t$, and the arguments of $\omega^{(n)}, \theta^{(n)}, u^{(n)}$, and $v^{(n)}$ are $x, \alpha t s$, and $t$.

For an arbitrary $\eta>0$ we have

$$
\begin{aligned}
& I_{1} \leq \eta \int_{0}^{1} \int_{\Omega} Q_{n}^{p-1}\left(\left|\nabla \omega^{(n)}\right|^{2}+\left|\nabla \theta^{(n)}\right|^{2}\right) d x d s+ \\
& \quad C_{\eta} \int_{0}^{1} \int_{\Omega} Q_{n}^{p-1}\left(\left(\omega^{(n)}\right)^{2}+\left(\theta^{(n)}\right)^{2}\right)|\alpha|^{2} s^{2} d x d s \leq \eta I_{0}+C_{\eta}|\alpha|^{2} \psi_{n}(t) .
\end{aligned}
$$

Next,

$$
I_{2}=\frac{1}{2 p} \int_{0}^{1} \int_{\Omega} \nabla Q_{n}^{p} \cdot u^{(n-1)} d x d s=0
$$

For $I_{3}$ we have

$$
I_{3} \leq \eta I_{0}+C_{\eta} \int_{0}^{1} \int_{\Omega} Q_{n}^{p}\left|v^{(n-1)}\right|^{2} d x d s \leq \eta I_{0}+C_{\eta} I_{3}^{\prime} I_{3}^{\prime \prime},
$$

where

$$
\begin{aligned}
I_{3}^{\prime} & =\left(\int_{0}^{1} \int_{\Omega} Q_{n}(x, s, t)^{p^{2} /(p-1)} d x d s\right)^{(p-1) / p}, \\
I_{3}^{\prime \prime} & =\sum_{j=1}^{2}\left(\int_{0}^{1} \int_{\Omega}\left|v_{j}^{(n-1)}(x, \alpha t s, t)\right|^{2 p} d x d s\right)^{1 / p} .
\end{aligned}
$$

We write $I_{3}^{\prime}$ as follows

$$
I_{3}^{\prime}=\left\|Q_{n}^{p / 2}\right\|_{L_{\beta}\left(\Omega_{0}\right)}^{2}, \quad \Omega_{0}=\Omega \times[0,1] \subset \mathbb{R}^{3}, \quad \beta=2 p /(p-1), \quad 2 \leq \beta \leq 6,
$$

and use in $\Omega_{0}$ the Gagliardo-Nirenberg inequality

$$
\|A\|_{L_{\beta}\left(\Omega_{0}\right)} \leq C\|A\|_{L_{2}\left(\Omega_{0}\right)}^{3 / \beta-1 / 2}\left\|\nabla_{x, s} A\right\|_{L_{2}\left(\Omega_{0}\right)}^{3 / 2-3 / \beta}+C\|A\|_{L_{2}\left(\Omega_{0}\right)}
$$


for $A=A(x, s)=Q_{n}^{p / 2}(x, s, t)$. We have

$$
\begin{gathered}
\left\|\nabla_{x, s} A\right\|_{L_{2}\left(\Omega_{0}\right)}^{2}=\left\|\nabla_{x, s} Q_{n}^{p / 2}\right\|_{L_{2}\left(\Omega_{0}\right)}^{2}= \\
p^{2} \int_{0}^{1} \int_{\Omega} Q_{n}^{p-2}\left(\left(\omega^{(n)} \nabla \omega^{(n)}+\theta^{(n)} \nabla \theta^{(n)}\right)^{2}+\right. \\
\left.t^{2}\left(\theta^{(n)} \alpha \cdot \nabla \omega^{(n)}-\omega^{(n)} \alpha \cdot \nabla \theta^{(n)}\right)^{2}\right) d x d s \leq \\
\leq C p^{2}\left(1+|\alpha|^{2} t^{2}\right) I_{0} .
\end{gathered}
$$

Hence,

$$
\left\|\nabla_{x, s} A\right\|_{L_{2}\left(\Omega_{0}\right)}^{3 / 2-3 / \beta}=\left\|\nabla_{x, s} Q_{n}^{p / 2}\right\|_{L_{2}\left(\Omega_{0}\right)}^{3 / 2 p} \leq C\left(1+|\alpha|^{2} t^{2}\right)^{3 / 4 p} I_{0}^{3 / 4 p} .
$$

Next, $\left\|Q_{n}^{p / 2}\right\|_{L_{2}\left(\Omega_{0}\right)}^{2}=\psi_{n}(t)$

$$
\|A\|_{L_{2}\left(\Omega_{0}\right)}^{3 / \beta-1 / 2}=\left\|Q_{n}^{p / 2}\right\|_{L_{2}\left(\Omega_{0}\right)}^{(2 p-3) / 2 p}=\psi_{n}(t)^{(2 p-3) / 4 p}
$$

and hence

$$
I_{3}^{\prime} \leq C\left(1+|\alpha|^{2} t^{2}\right)^{3 / 2 p} I_{0}^{3 / 2 p} \psi_{n}(t)^{(2 p-3) / 2 p}+C \psi_{n}(t) .
$$

We now consider $I_{3}^{\prime \prime}$. Since $v_{j}^{(n-1)}(x, 0, t)=0$ (the solution restricted to $y=0$ is real-valued), we have (using the Cauchy-Riemann equations for $v_{j}$ )

$$
\begin{aligned}
\left|v_{j}^{(n-1)}(x, \alpha t s, t)\right|= & \left|\sum_{k=1}^{2} \alpha_{k} t s \int_{0}^{1} \partial_{y_{k}} v_{j}^{(n-1)}(x, \alpha t s \tau, t) d \tau\right|= \\
& \left|\sum_{k=1}^{2} \alpha_{k} t s \int_{0}^{1} \partial_{k} u_{j}^{(n-1)}(x, \alpha t s \tau, t) d \tau\right| .
\end{aligned}
$$

Then

$$
\begin{array}{r}
I_{3}^{\prime \prime}=\sum_{j=1}^{2}\left(\int_{0}^{1} \int_{\Omega}\left|\sum_{k=1}^{2} \alpha_{k} t s \int_{0}^{1} \partial_{k} u_{j}^{(n-1)}(x, \alpha t s \tau, t) d \tau\right|^{2 p} d x d s\right)^{1 / p} \leq \\
C|\alpha|^{2} t^{2}\left(\int_{0}^{1} \int_{\Omega} \int_{0}^{1}\left|\nabla u^{(n-1)}(x, \alpha t s \tau, t)\right|^{2 p} s^{2 p} d \tau d x d s\right)^{1 / p}= \\
C|\alpha|^{2} t^{2}\left(\int_{0}^{1} s^{2 p} d s \int_{0}^{1} d \tau \int_{\Omega}\left|\nabla u^{(n-1)}(x, \alpha t s \tau, t)\right|^{2 p} d x\right)^{1 / p} .
\end{array}
$$

Since $u=\nabla^{\perp} \Delta^{-1} \omega$, we have (see $[\mathbf{2 2}],[\mathbf{4 4}]$ )

$$
\left(\int_{\Omega}|\nabla u(x)|^{2 p} d x\right)^{1 / 2 p}=\left\|\nabla \nabla^{\perp} \Delta^{-1} \omega\right\|_{L_{2 p}} \leq\left\|\Delta^{-1} \omega\right\|_{W_{2 p}^{2}} \leq C p\|\omega\|_{L_{2 p}} .
$$

Therefore

$$
\begin{array}{r}
I_{3}^{\prime \prime} \leq C p^{2}|\alpha|^{2} t^{2}\left(\int_{0}^{1} \int_{0}^{1} \int_{\Omega}\left|\omega^{(n-1)}(x, \alpha t s \tau, t)\right|^{2 p} d x s^{2 p} d s d \tau\right)^{1 / p} \leq \\
C p^{2}|\alpha|^{2} t^{2}\left(\int_{0}^{1} \int_{\Omega} Q_{n-1}^{p} d x d s\right)^{1 / p} \leq C p^{2}|\alpha|^{2} t^{2} \psi_{n-1}(t)^{1 / p},
\end{array}
$$


where we have used $\int_{0}^{1} \int_{0}^{1} h(s \tau) s^{2 p} d s d \tau \leq(2 p)^{-1} \int_{0}^{1} h(s) d s$. Combining this with (3.14) and (3.15) we obtain

$$
\begin{aligned}
& I_{3} \leq \eta^{\prime} I_{0}+C_{\eta^{\prime}} p^{2}|\alpha|^{2} t^{2}\left(1+|\alpha|^{2} t^{2}\right)^{3 / 2 p} I_{0}^{3 / 2 p} \psi_{n}(t)^{(2 p-3) / 2 p} \psi_{n-1}(t)^{1 / p}+ \\
& C p^{2}|\alpha|^{2} t^{2} \psi_{n-1}(t)^{1 / p} \psi_{n}(t) \leq \\
& \eta I_{0}+C_{\eta} p^{2}(|\alpha| t)^{4 p /(2 p-3)}\left(1+|\alpha|^{2} t^{2}\right)^{3 /(2 p-3)} \psi_{n-1}(t)^{2 /(2 p-3)} \psi_{n}(t)+ \\
& C p^{2}|\alpha|^{2} t^{2} \psi_{n-1}(t)^{1 / p} \psi_{n}(t) .
\end{aligned}
$$

Finally, we estimate $I_{4}$ :

$$
\begin{gathered}
\left.\left.I_{4} \leq \int_{0}^{1} \int_{\Omega} Q_{n}^{p-1}\left(\left(\omega^{(n)}\right)^{2}+\theta^{(n)}\right)^{2}\right) \eta \mu+\left(F^{2}+G^{2}\right) /(4 \eta \mu)\right) d x d s \leq \\
\eta I_{0}+C_{\eta}\left(M_{F}^{2} / \mu\right) \int_{0}^{1} \int_{\Omega} Q_{n}^{p-1} d x d s \leq \eta I_{0}+C_{\eta}\left(M_{F}^{2} / \mu\right) \psi_{n}(t)^{(p-1) / p},
\end{gathered}
$$

where $M_{F}$ is defined in (3.6), with the final estimate relying on $\Omega$ being $[0,1]^{2}$.

Taking $\eta>0$ sufficiently small we infer from (3.11), (3.12), (3.13), (3.16), (3.17)

$$
\begin{array}{r}
\partial_{t} \psi_{n}(t) \leq C p|\alpha|^{2} \psi_{n}(t)+C p^{3}|\alpha|^{4 p /(2 p-3)} t^{4 p /(2 p-3)} \psi_{n-1}(t)^{2 /(2 p-3)} \psi_{n}(t)+ \\
C p^{3}|\alpha|^{(4 p+6) /(2 p-3)} t^{(4 p+6) /(2 p-3)} \psi_{n-1}(t)^{2 /(2 p-3)} \psi_{n}(t)+ \\
C p^{3}|\alpha|^{2} t^{2} \psi_{n-1}(t)^{1 / p} \psi_{n}(t)+C p \psi_{n}(t)^{(p-1) / p} M_{F}^{2} / \mu,
\end{array}
$$

where

$$
\psi_{n}(0)=\int_{\Omega}\left(\omega_{0}(x)^{2}+\varepsilon\right)^{p} d x
$$

We set $\varphi_{n}(t)=\psi_{n}(t)^{1 / 2 p}$ and obtain the differential inequality for $\varphi_{n}$ :

$$
\begin{array}{r}
\partial_{t} \varphi_{n}(t) \leq C|\alpha|^{2} \varphi_{n}(t)+C p^{2}|\alpha|^{4 p /(2 p-3)} t^{4 p /(2 p-3)} \varphi_{n-1}(t)^{4 p /(2 p-3)} \varphi_{n}(t)+ \\
C p^{2}|\alpha|^{(4 p+6) /(2 p-3)} t^{(4 p+6) /(2 p-3)} \varphi_{n-1}(t)^{4 p /(2 p-3)} \varphi_{n}(t)+ \\
C p^{2}|\alpha|^{2} t^{2} \varphi_{n-1}(t)^{2} \varphi_{n}(t)+C \varphi_{n}(t)^{-1} M_{F}^{2} / \mu .
\end{array}
$$

We now use the Gronwall-type Lemma 3.3 from [33] below and see that $\varphi(t) \leq$ $2 \varphi(0)$ on the time interval specified in (3.18), (3.19), and letting $\varepsilon \rightarrow 0$ we obtain

$$
\int_{0}^{1} \int_{\Omega}\left(\omega^{(n)}(x, \alpha t s, t)^{2}+\theta^{(n)}(x, \alpha t s, t)^{2}\right)^{p} d x d s \leq 2^{2 p} M_{2 p}^{2 p}
$$

for $t \geq 0,|\alpha| t \leq \delta_{F}$ and

$$
t \leq \min \left(A_{1}, A_{2}, A_{3}, A_{4}, A_{5}\right)
$$


where

$$
\begin{aligned}
& A_{1}=\frac{1}{C|\alpha|^{2}}, \\
& A_{2}=\frac{1}{C p^{(4 p-6) /(6 p-3)}|\alpha|^{4 p /(6 p-3)} M_{2 p}^{4 p /(6 p-3)}}, \\
& A_{3}=\frac{1}{C p^{(4 p-6) /(6 p+3)}|\alpha|^{(4 p+6) /(6 p+3)} M_{2 p}^{4 p /(6 p+3)}}, \\
& A_{4}=\frac{1}{C p^{2 / 3}|\alpha|^{2 / 3} M_{2 p}^{2 / 3}}, \\
& A_{5}=\frac{M_{2 p}^{2}}{C M_{F}^{2} \mu^{-1}} .
\end{aligned}
$$

We now set

$$
t_{0}=\frac{M_{2 p}^{2}}{C M_{F}^{2} / \mu} .
$$

Then the condition

$$
t \leq \min \left(A_{1}, A_{2}, A_{3}, A_{4}\right)
$$

can be written in terms of $y=\alpha t$ as follows

$$
|y| \leq \min \left(\frac{t^{1 / 2}}{C}, \frac{1}{C p t^{(2 p-3) / 4 p} M_{2 p}}, \frac{1}{C p t^{(2 p-3) /(4 p+6)} M_{2 p}^{2 p /(2 p+3)}}, \frac{1}{p t^{1 / 2} M_{2 p}}\right) .
$$

Now for $t_{0}$ defined in (3.20) and

$$
\delta(t)=\min \left(\frac{t^{1 / 2}}{C}, \frac{1}{C p t^{(2 p-3) / 4 p} M_{2 p}}, \frac{1}{C p t^{(2 p-3) /(4 p+6)} M_{2 p}^{2 p /(2 p+3)}}, \frac{1}{p t^{1 / 2} M_{2 p}}, \delta_{F}\right)
$$

we have for $0<t \leq t_{0}$ and $|y| \leq \delta(t)$

$$
\int_{0}^{1} \int_{\Omega}\left(\omega^{(n)}(x, s y, t)^{2}+\theta^{(n)}(x, s y, t)^{2}\right) d x d s \leq 2^{2 p} M_{2 p}^{2 p}
$$

for all integer $n \geq 1$. Therefore for any $y \in \mathbb{R}^{2}$ with $|y|=1$ this gives that

$$
\int_{0}^{\delta(t)} \int_{\Omega}\left(\omega^{(n)}(x, s y, t)^{2}+\theta^{(n)}(x, s y, t)^{2}\right) d x d s \leq 2^{2 p} \delta(t) M_{2 p}^{2 p}
$$

and since $\int_{0}^{\delta} f(s y) d s \leq B,|y|=1$ implies $\int_{|y| \leq \delta} f(y) d y \leq 2 \pi \delta B$, we obtain

$$
\int_{|y| \leq \delta(t)} \int_{\Omega}\left(\omega^{(n)}(x, y, t)^{2}+\theta^{(n)}(x, y, t)^{2}\right) d x d s \leq 2 \pi 2^{2 p} \delta(t)^{2} M_{2 p}^{2 p} .
$$

This estimate is uniform in $n$ and as in [23], [33] we obtain the existence of an analytic solution of (3.4) with analyticity radius satisfying (3.22). The proof is complete. 
Lemma 3.3. (See [33].) Let $y_{n}(t) \in C^{1}[0, T]$ be a sequence of non-negative functions satisfying $y_{0}(t) \leq M$ for $0 \leq t \leq T$, and $y_{n}(0) \leq M$ for $n \geq 1$. Suppose that on the interval $0 \leq t \leq T$

$$
\partial_{t} y_{n}(t) \leq \sum_{j=1}^{N} K_{j} t^{\alpha_{j}} y_{n}(t)^{\beta_{j}} y_{n-1}(t)^{\gamma_{j}},
$$

where $K_{j}>0, \alpha_{j}>-1, \beta_{j} \in \mathbb{R}$, and $\gamma_{j} \geq 0$ are given constants. Then $y_{n}(t) \leq 2 M$ for all $n=0,1,2, \ldots$ provided that

$$
0 \leq t \leq \min \left(T, \min _{j=1, \ldots, N}\left(\frac{\alpha_{j}+1}{N K_{j} 2^{\beta_{j}^{+}+\gamma_{j}} M^{\beta_{j}+\gamma_{j}-1}}\right)^{1 /\left(\alpha_{j}+1\right)}\right),
$$

where $\beta^{+}=\max (\beta, 0)$.

We can now state the main result of this section.

THEOREM 3.1. The solutions on the 2D space-periodic damped-driven NavierStokes system (1.6) lying on the global attactor $\mathcal{A}$ are analytic with space analyticity radius $l_{a}$ satisfying the lower bound

$$
l_{a} \geq \frac{|\Omega|^{1 / 2}}{C D^{1 / 2}(1+\log D)^{1 / 2}}, \quad \text { where } \quad D=\frac{\|\operatorname{rot} f\|_{\infty}|\Omega|}{\mu \nu} .
$$

Proof. We first observe that (3.23) is equivalent to the estimate

$$
l_{a} \geq \frac{1}{C D^{1 / 2}(1+\log D)^{1 / 2}}
$$

for the equation written in dimensionless form.

Next, by Young's inequality

$$
\begin{aligned}
& p t^{(2 p-3) / 4 p} M \leq C p M^{4 p /(4 p-3)} t^{1 / 2}+t^{-1 / 2}, \\
& p t^{(2 p-3) /(4 p+6)} M^{2 p /(2 p+3)} \leq C p M t^{1 / 2}+t^{-1 / 2} .
\end{aligned}
$$

Hence, the estimate (3.22) can be written as follows

$$
\delta(t) \geq \min \left(\frac{t^{1 / 2}}{C}, \frac{1}{C p t^{1 / 2}\left(M_{2 p}^{4 p /(4 p-3)}+M_{2 p}\right)}, \delta_{F}\right) .
$$

The solutions lying on the attractor are bounded in $L_{2 p}$ :

$$
\|\omega(t)\|_{L_{2 p}} \leq M_{2 p}, \quad \mathrm{M}_{2 p} \leq C M_{\infty}
$$

Setting

we see that

$$
p=C\left(1+\log M_{\infty}\right)
$$

$$
\left.p\left(M_{2 p}^{4 p /(4 p-3)}+M_{2 p}\right)\right|_{p=C\left(1+\log M_{\infty}\right)} \leq C\left(1+\log M_{\infty}\right) M_{\infty}
$$

and therefore

$$
\delta(t) \geq \min \left(\frac{t^{1 / 2}}{C}, \frac{1}{C\left(1+\log M_{\infty}\right) M_{\infty} t^{1 / 2}}, \delta_{F}\right) .
$$

At the moment of time

$$
t^{*}=\frac{1}{C\left(1+\log M_{\infty}\right) M_{\infty}},
$$


which for sufficiently large $M_{\infty}$ (the case of our interest) is smaller than $t_{0}$ defined in (3.20) (the details are given below) we have

$$
\delta\left(t^{*}\right) \geq \frac{1}{C M_{\infty}^{1 / 2}\left(1+\log M_{\infty}\right)^{1 / 2}} .
$$

Since $M_{\infty} \leq D($ see $(3.5))$, it follows that

$$
\delta\left(t^{*}\right) \geq \frac{1}{C D^{1 / 2}(1+\log D)^{1 / 2}} .
$$

By the invariance property of the attractor we see that on the attractor the above estimate holds for all $t^{*}$, which proves (3.24).

To complete the proof it remains to show that

$$
\frac{1}{C(1+\log D) D}=t^{*} \leq t_{0}=\frac{D^{2}}{C M_{F^{\prime}}^{2} / \mu^{\prime}}
$$

where in the expression for $t_{0}$ we reverted to the prime notation for the dimensionless damping coefficient $\mu^{\prime}$ and the forcing $F^{\prime}$. We relate the forcing term and its analytic extension by the equality

$$
M_{F^{\prime}}=K\left\|\operatorname{rot}^{\prime} f^{\prime}\right\|_{\infty}, \quad K=K\left(F, \delta_{F}\right) .
$$

Recalling that $f^{\prime}=\left(L^{3} / \nu^{2}\right) f, \mu^{\prime}=\left(L^{2} / \nu\right) \mu$, and $x^{\prime}=(1 / L) x$ we see that

$$
t_{0}=\frac{\nu}{C K^{2} \mu L^{2}} \text {. }
$$

Hence, (3.26) goes over to the condition

$$
C(1+\log D) \geq \frac{K^{2} \mu^{2}}{\|\operatorname{rot} f\|_{\infty}},
$$

which is obviously satisfied for all sufficiently small $\nu>0$. The proof is complete.

\section{Concluding remarks}

We consider the 2D space-periodic damped-driven Navier-Stokes system, that is, the Stommel-Charney barotropic model of ocean circulation without rotation. We have shown that the solutions lying on the attractor of this system with analytic forcing have space analyticity radius which up to a logarithmic term coincides with the small scale estimates both in terms of the sharp bounds for the fractal dimension of the global attractor, and in terms of the spatial lattice of determining nodes. The derivation of this lower bound for the analyticity radius essentially uses the techniques developed in $[\mathbf{3 3}]$.

\section{Acknowledgments}

We are grateful to the anonymous referee for the useful comments.

A.A.I. would like to thank the Mathematics Department at the University of California, Irvine, for their warm hospitality during the term of this project.

This work was supported in part by the US Civilian Research and Development Foundation, grant no. RUM1-2654-MO-05 ( A.A.I. and E.S.T.), by the Russian Foundation for Fundamental Research, grants nos. 06-01-00096 and 05-01-00429, and by the RAS Programme no.1 'Modern problems of theoretical mathematics' 
(A.A.I.). The work of E.S.T. was supported in part by the National Science Foundation, grant no. DMS-0504619, the ISF grant no. 120/6, and the BSF grant no. 2004271

\section{References}

1. Babin A.V., Vishik M.I. Attractors of Evolution Equations, Nauka, Moscow (1988) English transl. North-Holland, Amsterdam (1992).

2. Bardos C., Benachour S. Domaine d'analycité des solutions de l'equation d'Euler dans un ouvert de $\mathbb{R}^{n}$. Ann. Scuola Norm. Sup. Pisa 4, 647-687 (1977).

3. Chae D. On the ensemble average in the study of approximate inertial manifolds, II. J. Math. Anal. Appl. 164, 337-349 (1992).

4. Charney J.G. The Gulf stream as an inertial boundary layer. Proc. Nat. Acad. Sci. USA 41, 731-740 (1955).

5. Barcilon V., Constantin P., Titi E.S. Existence of solutions to the Charney model of the Gulf Stream. SIAM J. Math. Anal. 19, 1355-1364 (1988).

6. Constantin P., Foias C. Navier-Stokes Equations, Univ. of Chicago Press, Chicago (1988).

7. Constantin P., Foias C., Temam R. Attractors Representing Turbulent Flows. Mem. Amer. Math. Soc. 53, Providence, RI (1985).

8. Constantin P., Foias C., Temam R. On the dimension of the attractors in two-dimensional turbulence. Physica D 30, 284-296 (1988).

9. Doering C.R., Gibbon J.D. Applied Analysis of the Navier-Stokes Equations, Cambridge, Cambridge University Press (1997).

10. Dymnikov V.P., Filatov A.N. Mathematics of Climate Modelling, Boston, Birkhäuser (1997).

11. Doering C.R., Titi E.S. Exponential decay rate of the power spectrum for solutions of the Navier-Stokes equations. Phys. Fluids 7:6, 1384-1390 (1995).

12. Foias C., Jolly M.S., Manley O.P., Rosa R. On the Landau-Lifschitz degrees of freedom in 2-D turbulence. J. Stat. Phys. 111: 3/4, 1017-1019 (2003).

13. Foias C., Manley O., Rosa R., and Temam R. Navier-Stokes Equations and Turbulence, Cambridge, UK, Cambridge Univ. Press (2001).

14. Foias C., Manley O., Temam R. Modelling of the interaction of small and large eddies in two dimensional turbulent flows. Math. Mod. Num. Anal. 22, 93-118 (1988).

15. Foias C., Manley O., Temam R. Bounds for the mean dissipation of 2-D enstrophy and 3-D energy in turbulent flows. Physics Letters A 174, 210-215 (1993).

16. Foias C. and Prodi G. Sur le comportement global des solutions non stationnaires des équations de Navier-Stokes en dimension two. Rend. Sem. Mat. Univ. Padova 39, 1-34 (1967).

17. Foias C., Temam R. Gevrey class regularity for the solutions of the Navier-Stokes equations. J. Func. Anal. 87, 359-369 (1989).

18. Foias C., Temam R. Determination of the solutions of the Navier-Stokes equations by a set of nodal values. Mathematics of Computation 43, 117-133 (1984).

19. Friz P.K., Kukavica I., Robinson J.C. Nodal parameterizing of analytic attractors. Disc. Contin. Dynam. Syst. 7, 643-657 (2001).

20. Friz P.K., Robinson J.C. Parameterizing the attractor of the two-dimensional Navier-Stokes system with a finite number of nodal values. Physica D 148, 201-220 (2001).

21. Gibbon J.D., Titi E.S. Attractor dimension and small length scale estimates for the threedimensional Navier-Stokes equations. Nonlinearity 10, 109-119 (1997).

22. Gilbarg D., Trudinger N.S. Elliptic Partial Differential Equations of Second Order. 2nd edition. Springer-Verlag, Berlin, (1983).

23. Grujić Z., Kukavica I. Space analyticity for the Navier-Stokes and related equations with initial data in $L^{p}$. J. Func. Anal. 152, 447-466 (1998).

24. Hauk S. The Long-term Behavior of the Stommel-Charney Model of the Gulf Stream, an Analytical and Computational Study, Ph.D. Thesis, Department of Mathematics, University of California, Irvine (1997).

25. Henshaw W.D., Kreiss H.-O., Reyna L.G. Smalles scale estimates for the Navier-Stokes equations for incompressible fluids. Arch. Rat. Mech. Anal. 112, 21-44 (1990).

26. Ilyin A.A. The Euler equations with dissipation. Mat. Sbornik 182, no. 12, 1729-1739 (1991) English transl. in Math. USSR, Sbornik 74, no. 2, 475-486 (1993). 
27. Ilyin A.A., Miranville A., Titi E.S. A small viscosity sharp estimate for the global attractor of the 2-D damped-driven Navier-Stokes equations. Commun. Math. Sciences 2:3, 403-426 (2004).

28. Ilyin A.A., Titi E.S. Sharp estimates for the number of the degrees of freedom for the Dampeddriven 2-D Navier-Stokes equations. J. Nonlinear Sci. 16, 233-253 (2006).

29. Ilyin A.A., Titi E.S. The damped-driven 2D Navier-Stokes system on large elongated domains. Journal of Mathematical Fluid Mechanics 9, 1-17 (2007).

30. Jones D., Titi E.S. Upper bounds on the number of determining modes, nodes, and volume elements for the Navier-Stokes equations. Indiana Univ. Math. J. 42, 875-887 (1993).

31. Kato T. Strong $L^{p}$-solutions of the Navier-Stokes equation in $\mathbb{R}^{m}$, with applications to weak solutions. Math. Z. 187, , 471-480 (1984).

32. Kraichnan R.H. Inertial ranges in two-dimensional turbulence. Phys. Fluids 10, 1417-1423 (1967).

33. Kukavica I. On the dissipative scale for the Navier-Stokes equations. Indiana Univ. Math. J. 48, 1057-1081 (1999).

34. Ladyzhenskaya O.A. The Mathematical Theory of Viscous Incompressible Flow, Nauka, Moscow (1970); English transl. Gordon and Breach, New York (1969).

35. Liu V.X. A sharp lower bound for the Hausdorff dimension of the global attractors of the 2D Navier-Stokes equations. Comm. Math. Phys. 158 (1993), 327-339.

36. Oliver M., Titi E.S. On the domain of analyticity for solutions of second order analytic nonlinear differential equations. J. Differential. Equations. 174, 55-74 (2001).

37. Pedlosky J. Geophysical Fluid Dynamics, New York, Springer (1979).

38. Robinson J.C. Infinite Dimensional Dynamical Systems, Cambridge, Cambridge University Press, 2001.

39. Stommel H. The westward intensification of wind-driven ocean currents. Trans. Amer. Geophys. Union 29, 202-206 (1948).

40. Temam R. Navier-Stokes Equations. Theory and Numerical Analysis, Amsterdam, NorthHolland (1984).

41. Temam R. Infinite Dimensional Dynamical Systems in Mechanics and Physics, 2nd ed., New York, Springer-Verlag (1997).

42. Titi E.S. On a criterion for locating stable stationary solutions to the Navier-Stokes equations. Nonlin. Anal. 11:9, 1085-1102 (1987).

43. Wolansky G. Existence, uniqueness, and stability of stationary barotropic flow with forcing and dissipation. Comm. Pure Appl. Math 41, 19-46 (1988).

44. Yudovich V.I. On a bound for the solution of an elliptic equation. (Russian). Uspehi Mat. Nauk 20:2, 213-219 (1965).

Keldysh Institute of Applied Mathematics, Russian Academy of Sciences, Miusskaya SQ. 4, 125047 Moscow, Russia

E-mail address: ilyin@keldysh.ru

Department of Mathematics and Department of Mechanical and Aerospace Engineering, University of California, Irvine, California 92697, USA. Also: Department of Computer Science and Applied Mathematics, Weizmann Institute of Science, P.O. Box 26, Rehovot, 76100, IsRael

E-mail address: etiti@math.uci.edu, also: edriss.titi@weizmann.ac.il 